\title{
Radiofrequency Ablation Combined with Resection Enhances Chance for Curative Treatment of Hepatocellular Carcinoma
}

\author{
Ronnie T. P. Poon, MS, PhD, FRCS (Edin), FACS
}

Departments of Surgery, The University of Hong Kong, Queen Mary Hospital, 102 Pokfulam Road, Hong Kong, China

Hepatic resection is the mainstay of curative treatment for hepatocellular carcinoma (HCC), with perioperative and survival results improving in recent years. ${ }^{1}$ Radiofrequency ablation (RFA) is a rapidly growing thermal ablative therapy for small HCC that is also potentially curative. Prospective randomized trials have shown that RFA is superior to ethanol injection in inducing complete tumor necrosis, resulting in better long-term progression-free and overall survival. ${ }^{2,3}$ Furthermore, a recent randomized trial suggested that RFA might achieve similar longterm survival results compared with resection for solitary $\mathrm{HCC}<5 \mathrm{~cm}$ in diameter, although more data from further randomized trials are needed to provide better evidence. ${ }^{4}$ Although RFA is competitive with resection in the treatment of a small solitary HCC, RFA and resection could be complementary to each other in the management of multifocal HCCs. Combined hepatectomy and radiofrequency ablation for multifocal hepatocellular carcinomas: long-term follow-up results and prognostic factors Choi et al. ${ }^{5}$ reported favorable perioperative results and longterm survival in a series of 53 patients who had undergone combined resection and ablation for multifocal HCCs. This study demonstrated an important role for RFA in increasing the chance of curative treatments for patients with multifocal tumors that may otherwise be considered unresectable.

Received July 9, 2007; accepted July 10, 2007; published online: September 25, 2007.

Address correspondence and reprint requests to: Ronnie T. P. Poon, MS, PhD, FRCS (Edin), FACS; E-mail: poontp@ hkucc.hku.hk

Published by Springer Science+Business Media, LLC $\odot 2007$ The Society of Surgical Oncology, Inc.
The role of combined resection and ablation has been previously reported in patients with colorectal liver metastasis. ${ }^{6}$ Combined resection and RFA may play an even more important role in the management of HCC because of the high frequency of multifocal tumors and associated cirrhosis, which limits the applicability of extended resection to encompass multifocal or bilobar HCCs. A previous study of patients with bilobar HCCs diagnosed after laparoscopy or laparotomy has shown that combined resection of HCC in one lobe and wedge resection or ethanol ablation of lesions in the contralateral lobe greatly prolonged the long-term survival compared with nonsurgical treatments. ${ }^{7}$ However, wedge resection for contralateral lobe lesions can only be performed for superficial tumors, and ethanol injection therapy can only achieve a complete necrosis of approximately $70 \%$, even for tumors $<3 \mathrm{~cm}$. RFA is a better modality for treatment of the contralateral lesions and could even be used for deep-seated lesions. The strategy of RFA combined with resection is particularly useful for patients with a large tumor in one lobe and one or two smaller tumor nodules in the contralateral lobe, for which neither resection or RFA alone is adequate for treatment.

Although the strategy of combined RFA and resection may reduce the surgical risk compared with an extended hepatectomy aimed to encompass multiple tumors, it is still a high-risk procedure in patients who have limited liver function reserve. Choi et al. reported no operative mortality and an $8 \%$ major complication rate, which are indeed very favorable results. However, it should be noted that their selection criterion for lobectomy of $<10 \%$ indocyanine green 
retention at 15 minutes is quite restrictive. In my center, the selection criterion for major liver resection has been extended to cirrhotic patients with indocyanine green retention at 15 minutes up to $20 \% .{ }^{8}$ It is possible that some of the patients with multifocal tumors treated with RFA combined with resection in the study of Choi et al. could have been treated by extended hepatic resection. None of the patients in the study had received right portal vein embolization before surgery. In patients with mild cirrhosis, right portal vein embolization is effective in inducing hypertrophy of the left lateral segment and may allow safe resection of multifocal tumors confined to right lobe and segment $4 .{ }^{9}$ The role of combined RFA and resection is more important in patients with marked cirrhosis and truly borderline liver function. The safety of RFA combined with major resection in such patients remains to be evaluated.

The 5-year survival rate of $55 \%$ after combined resection and RFA in the study of Choi et al. is encouraging. Of a total of 66 tumors ablated, there was only one incomplete ablation and another two with local tumor progression after a median followup of 22 months in the whole group. However, the 5year cancer-free survival was $0 \%$, indicating that tumor recurrence at other parts of the liver or extrahepatic sites is a major problem. The presence of multifocal tumors implies either a high propensity for multicentric carcinogenesis or intrahepatic metastasis from a dominant tumor. A high recurrence rate in patients with multifocal tumors after combined RFA and resection is expected. Thus far, there is no wellestablished adjuvant therapy that could reduce tumor recurrence after resection or RFA. To further improve the long-term outcome, it is imperative that more research effort is devoted to the study of novel adjuvant therapies for HCC.

Although the study of Choi et al. demonstrated favorable results of RFA combined with resection for multifocal HCCs, it remain unclear to what extent this could increase the chance of curative treatment for tumors otherwise not considered curable. The effect of RFA in this aspect could only be clarified by comparing the management of multifocal HCCs before and after availability of RFA. Further studies should also compare the outcome of RFA combined with resection with that of patients with resection alone to see whether the survival results are truly comparable. Nonetheless, RFA combined with resection should be considered the treatment of choice for multifocal HCCs that are otherwise not amenable to resection or liver transplantation.

\section{REFERENCES}

1. Poon RT, Fan ST, Lo CM, et al. Improving survival results after resection of hepatocellular carcinoma: a prospective study of 377 patients over 10 years. Ann Surg 2001; 234:63-70.

2. Shiina $\mathrm{S}$, Teratani $\mathrm{T}$, Obi $\mathrm{S}$, et al. A randomized controlled trial of radiofrequency ablation with ethanol injection for small hepatocellular carcinoma. Gastroenterology 2005; 129:122-30.

3. Lin SM, Lin CJ, Lin CC, et al. Radiofrequency ablation improves prognosis compared with ethanol injection for hepatocellular carcinoma $<$ or $=4 \mathrm{~cm}$. Gastroenterology 2004; 127:1714-23.

4. Chen MS, Li JQ, Zheng Y, et al. A prospective randomized trial comparing percutaneous local ablative therapy and partial hepatectomy for small hepatocellular carcinoma. Ann Surg 2006; 243:321-8.

5. Choi D, Lim HK, Joh JW, et al. Combined hepatectomy and radiofrequency ablation for multifocal hepatocellular carcinomas: long-term follow-up results and prognostic factors. Ann Surg Oncol 2007; 14: doi: 10.1245/s10434-007-9492-7 [Online July 26, 2007].

6. Pawlik TM, Iso F, Cohen DS, et al. Combined resection and radiofrequency ablation for advanced hepatic malignancies: results in 172 patients. Ann Surg Oncol 2003; 10:1059-69.

7. Liu CL, Fan ST, Lo CM, et al. Hepatic resection for bilobar hepatocellular carcinoma: is it justified? Arch Surg 2003; 138:100-4.

8. Poon RT, Fan ST.. Hepatectomy for hepatocellular carcinoma: patient selection and postoperative outcome. Liver Transpl 2004; 10(2 Suppl 1):S39-45.

9. Ogata S, Belghiti J, Farges O, et al. Sequential arterial and portal vein embolizations before right hepatectomy in patients with cirrhosis and hepatocellular carcinoma. Br J Surg 2006; 93:1091-8. 